\title{
PyCOMPSs: Parallel Computational Workflows in Python
}

\author{
Enric Tejedor • Yolanda Becerra • Guillem Alomar • Anna Queralt • \\ Rosa M. Badia • Jordi Torres · Toni Cortés · Jesús Labarta
}

Received: date / Accepted: date

\begin{abstract}
The use of the Python programming language for scientific computing has been gaining momentum in the last years. The fact that it is compact and readable and its complete set of scientific libraries are two important characteristics that favour its adoption. Nevertheless, Python still lacks a solution for easily parallelising generic scripts on distributed infrastructures, since the current alternatives mostly require the use of APIs for message passing or are restricted to embarrassingly-parallel computations.
\end{abstract}

In that sense, this paper presents PyCOMPSs, a framework that facilitates the development of parallel computational workflows in Python. In this approach, the user programs her script in a sequential fashion and decorates the functions to be run as asynchronous parallel tasks. A runtime system is in charge of exploiting the inherent concurrency of the script, detecting the data dependencies between tasks and spawning them to the available resources.

Furthermore, we show how this programming model can be built on top of a big data storage architecture, where the data stored in the backend is abstracted and

E. Tejedor, Y. Becerra, G. Alomar, A. Queralt, R. M. Badia, J. Torres, T. Cortés, J. Labarta

Department of Computer Sciences,

Barcelona Supercomputing Center (BSC-CNS)

Barcelona - Spain

E-mail: enric.tejedor@bsc.es, yolanda.becerra@bsc.es, guillem.alomar@bsc.es, anna.queralt@bsc.es

jordi.torres@bsc.es, toni.cortes@bsc.es

jesus.labarta@bsc.es

R. M. Badia

Artificial Intelligence Research Institute (IIIA),

Spanish Council for Scientific Research (CSIC)

Barcelona - Spain

E-mail: rosa.m.badia@bsc.es accessed from the application in the form of persistent objects.

Keywords Scientific Computing · Parallel Programming Models · Python · Big Data Storage

\section{Introduction}

The use of the Python programming language for scientific computing has been gaining momentum in the last years, sometimes replacing traditional tools such as Matlab [8]. The fact that Python is free software makes it available to anyone at no cost, and its portability enables execution on a variety of platforms. The language itself is compact, readable and very suited for rapid prototyping, while being powerful enough for writing large applications. Even though some people do not consider it efficient enough, Python integrates very well with $\mathrm{C} / \mathrm{C}++$, allowing to easily invoke external modules programmed in those languages for the sake of performance or code reuse. Furthermore, it has a rich set of scientific libraries, e.g. for numeric computation [9], data processing and analysis [17,10], plotting [7] and graphical interfaces [6].

All these advantages make Python appealing to the scientific community but, for the language to be used in big projects, it needs to be parallelisable. Its default and most widely-used implementation, CPython [15], cannot run multiple threads at once because of a global lock in the interpreter. In response, a number of alternatives for spawning multiple Python processes have appeared [11], both for shared-memory environments and for distributed infrastructures such as grids, clusters and clouds, as will be discussed in Section 2. However, most of these solutions are MPI wrappers, only permit to launch embarrassingly parallel computations 
or require the user to include infrastructure-related details in the application.

In that sense, this paper presents PyCOMPSs, a parallel programming framework for Python applications that overcomes the aforementioned limitations of other approaches. PyCOMPSs is built on top of COMPSs $[30,28]$ and it aims to facilitate the development of computational workflows in Python for distributed infrastructures. For that purpose, it offers a programming model based on sequential development - the application is a plain sequential Python script - where the user annotates the functions to be run as asynchronous parallel tasks. A runtime system is in charge of automatically exploiting the inherent concurrency of the script, detecting the data dependencies between tasks and spawning them to the available resources.

Furthermore, for PyCOMPSs scripts to easily access and compute on huge data sets, the programming model has been implemented on top of a big data storage platform. In our proposal, the application sees such big data in the form of regular Python objects that can be made persistent. The objective here is twofold: first, make possible for a Python script to handle big objects (too big to fit in the memory of a single node) and, second, offer a simple mechanism to access those objects and manage their persistency. Thus, Python programs can keep and share the modifications made to objects, with no need to explicitly read/write the data to persistent storage (e.g. a file); the actual access to the data is kept transparent to the programmer, who uses the references to the objects to operate with them in a normal way. By placing PyCOMPSs on top of such a storage platform, we intend to integrate the automatic detection and exploitation of parallelism in Python scripts with a data model that targets both big data and persistency.

The paper is structured as follows. Section 2 discusses the related work. Section 3 provides an overview of the PyCOMPSs programming model. Section 4 describes the basic runtime implementation of the model. Section 5 explains how PyCOMPSs has been integrated in a big data storage platform. Section 6 presents the results of the experiments. Finally, the conclusions and future work can be found in Section 7 .

\section{Related Work}

Although Python distributions include a threading module, there is not a simple way for parallelising applications. Although several threads can be used, the most popular implementation (CPython) does not support thread concurrency and the Global Interpreter Lock
(GIL) does not enable more than one thread to execute Python bytecode at a time. In [11] the alternatives available for parallelising Python applications are classified according to the platform that is tackled: Symmetric Multiprocessing (SMP), Cluster Computing, Cloud Computing and Grid Computing.

The Python multiprocessing module [14] supports the spawning of processes in SMP machines using an API similar to the threading module, with explicit calls to create processes, argument passing, execution, synchronization, result collection, etc. The multiprocessing module avoids the GIL issue by using subprocesses initiated with a fork system call instead of threads.

Parallel Python (PP) [12] is a Python module which provides mechanisms for parallel execution of Python code on SMP and clusters. It is based on an API which provides explicit functions to specify the number of workers to be used, submit the jobs for execution, get the results from the workers, etc. Similar to the multiprocessing module, all the management of the parallelism is delegated to the programmer, mixing the actual algorithm with the parallelism management.

In [29] the Pool/Map approach is described, as well as the Process/Queue approach. The Pool/Map approach spawns a pool of worker processes and returns a list of results. The Python map function is extended to the multiprocessing module and can be used with the Pool class to instantiate with a single operation a set of worker processes that will work in parallel and collect the results, using the regular Python syntax. The Process/Queue approach again extends the multiprocessing module to enable more than one input parameter to the concurrent function. This approach creates two FIFO queues, one for the input data and one for receiving output data. The parallel worker processes are started using the Process class. In the same paper, the Pool/Map, Process/Queue approach and Parallel Python are compared when solving parallel astronomical data processing applications, the Process/Queue approach showing better perfomance. Although in terms of features, Parallel Python will also enable execution in clusters, while the approaches based in multiprocessing can only be executed in a single node.

Other approaches to implement parallelism with Python are based on MPI wrappers. MPI for Python (mpi4py) [22] provides bindings of the Message Passing Interface (MPI) standard for the Python programming language, allowing any Python program to exploit multiple processors. It supports point-to-point and collective communications of any picklable Python object, as well as optimized communications of Python object exposing the single-segment buffer interface. A similar approach is followed by pyMPI [13]. 
dispy [4] is a Python framework for parallel execution of computations by distributing them across multiple processors in a single machine (SMP), among many machines in a cluster, grid or cloud. It is based on an API that enables to explicitly create jobs, submit them for execution, execute callbacks on job completion, wait for finalization, etc.

Alternatives to flow-based programming in Python are described in [5]. Ruffus [26] offers a syntax to explicitly define computational pipelines in Python using decorators. Cosmos [25] is another alternative to implement workflows in Python using the MapReduce paradigm.

Celery [2] also uses decorators to implement an asynchronous invocation of tasks in a server. The execution units are tasks, that are executed concurrently on a single or more worker web servers using multiprocessing. Tasks can be executed asynchronously or synchronously as invocations to a web service.

With regard these previous alternatives, PyCOMPSs provides an approach to parallelize codes without the need of expressing explicitly the concurrency and to execute them in distributed platforms, including SMPs, clusters and clouds. Ruffus, as PyCOMPSs, it is based on the use of decorators, although the decorators in Ruffus explicitly tell how the task functions are connected between them and the data exchanged between the nodes of the pipelines are always files, while PyCOMPSs is also more flexible in this aspect, giving support to regular Python objects. Ruffus uses the Python multiprocessing module to create one process per task in the pipeline. While Ruffus also provides a parallel construct, it is totally explicit, while in PyCOMPSs the parallelism is implicit, automatically detected by the runtime.

When comparing PyCOMPSs with previous work, it provides a generic (non ad-hoc solution) with a simple and flexible interface. The codes are programmed in native Python, with decorators used to indicate which parts of the code should be instantiated as tasks and to give hints about the arguments' directionality which will be used by the PyCOMPSs runtime to automatically find data dependences between tasks. The API of PyCOMPSs is composed of a single function that it is used to synchronize the completion of tasks at the end of parallel sections.

PyCOMPSs enables programming in parallel in the closest way to sequential code with the minimum number of references in the code to computational and data resources and concurrency. Also, the concurrency that it is supported is not only fork-join or MapReduce, but more flexible parallelism described in tasks' graphs that are dynamically built at execution time.
In the same way that PyCOMPSs abstracts concurrency from applications, access to distributed persistent objects is also transparent to the programmer thanks to our persistent storage layer. Current solutions that support persistent objects in Python provide an intuitive way to manage them from applications. Some examples are object databases such as ZODB [20], or the ORM libraries SQLAlchemy [18] or SQLObject [19]. However, they do not implement the functionality that allows PyCOMPSs to exploit data locality, which will be detailed in section 5. In particular, they do not provide the information that PyCOMPSs requires to schedule tasks next to the objects they manipulate. Additionally, our storage layer also provides the abiltiy to iterate on persistent dictionaries both expoiting data locality and parallelism.

Another approach to develop programmatic workflows is Swift, which is based in its own scripting language and finds the opportunities for parallel execution as a combination of parallel loop constructs and an implicit data-flow programming model [31]. As data types that are handled, Swift tasks only can manage files. Previously to this work, GRIDSs (the predecessor of COMPSs) was proposed for workflows described in $\mathrm{C} / \mathrm{C}++[21]$ also being able to unveal task parallelism through the creation at runtime of a data-dependence task graph defined by files as data objects.

\section{PyCOMPSs Programming Model}

PyCOMPSs is a programming framework that aims to facilitate the parallelisation of Python scripts. For that purpose, it offers a simple programming model based on sequential development: a PyCOMPSs application is a plain sequential Python script. In the model, the user is mainly responsible for (i) identifying the functions to be executed as asynchronous parallel tasks and (ii) annotating them with a standard Python decorator. A runtime system is in charge of exploiting the inherent concurrency of the script, automatically detecting and enforcing the data dependencies between tasks and spawning these tasks to the available resources, which can be nodes in a cluster, cloud or grid.

Section 3.1 will present a first example of a Python script parallelised with PyCOMPSs, while Section 3.2 will provide a more comprehensive specification of the PyCOMPSs programming model syntax and options.

\subsection{First Example}

The first step when programming with PyCOMPSs consists in defining the tasks of the application. A Python 
script may be composed by calls to multiple functions, and some of them may be computationally intensive. Such functions may be good candidates to be defined as tasks, so that they can be executed in parallel on a set of distributed resources.

As an example, let us consider the code in Figure 1(a). This script performs some computation for a number of steps (line 5) and merges the partial results, of type dictionary, into a final dictionary (line 6, variable result). All the computations receive a configuration parameter initialised in line 3 . The script can be executed as a sequential Python program but, in order to parallelise it with PyCOMPSs, we will define as tasks three functions called by the script: init_conf, compute_step and merge.

Task definition in PyCOMPSs is done by means of Python decorators [3], which are part of the standard Python syntax and permit to wrap calls to functions and with some additional behaviour. In particular, the user needs to add, before the definition of the function, a @task decorator that describes the task. Continuing with the example, Figure 1(b) shows the code of the aforementioned functions together with their @task decorators. Function init_conf returns an object of class Configuration (defined in line 1), as stated by its decorator (line 4). Similarly, compute_step returns a dictionary (as specified in the decorator of line 7) and receives two parameters: an integer and a Configuration object. Finally, merge receives two dictionary parameters and merges them into the first one (line 13); in order to state that the first dictionary will be modified inside the task, the decorator defines it as an input-output parameter (line 11).

Once the functions intended to be tasks are properly decorated, a Python script is ready to be executed with PyCOMPSs. When running the script, PyCOMPSs creates an asynchronous task for each invocation to a decorated function, adding these tasks to a dependency graph. The nodes of such graph are the tasks, and the edges represent their data dependencies. In order to detect data dependencies between tasks, PyCOMPSs uses the information about parameter direction specified in the @task decorator (e.g. INOUT for the dict1 parameter of function merge in Figure 1(b)). For instance, if a task writes some data and a subsequent task reads the same data, there is a data dependency between these tasks. Data dependencies are automatically enforced by PyCOMPSs to ensure the correct execution of the application.

In that sense, Figure 2 depicts the task dependency graph built on the fly by PyCOMPSs for the example in Figure 1(a). The first asynchronous task that is created corresponds to function init_conf, and after that

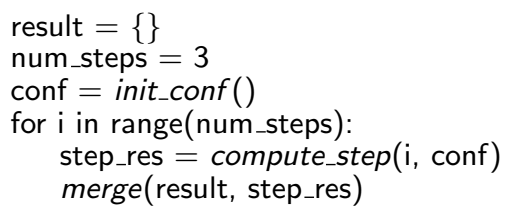

(b)

Fig. 1 Example of a sequential Python script parallelised with PyCOMPSs: (a) main program of the script, (b) task definition.

the main program proceeds immediately to execute the loop of computation and merge tasks. Inside the loop, a total of 3 compute_step tasks are generated, and they all depend on the previous init_conf task because they receive the configuration object conf as input parameter if no direction is specified for a parameter, it defaults to IN. The loop also generates 3 merge tasks, each depending on their corresponding compute_step for the partial result of the iteration (variable step_res); moreover, each merge task depends on the result produced by the previous iteration (stored in result) and, consequently, they are arranged in a chain of tasks.

Once the loop is completely unrolled, the program reaches lines 7-9, where the final result in variable result is printed. Before printing it, though, the script needs to synchronise for the last value of result, produced by the last merge task. In order to do that, PyCOMPSs provides an API function, compss_wait_on, which stalls the main control flow until the last value of result is obtained. Hence, the call to compss_wait_on in line 8 will wait for the last merge task to finish before getting and returning the final result, so that it can be printed in line 9 .

It is important to note how PyCOMPSs is able to detect and exploit the inherent concurrency of the 


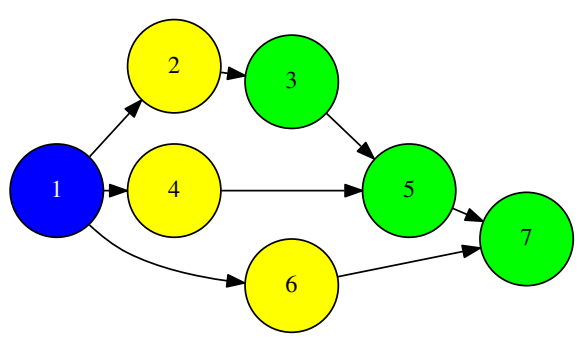

Fig. 2 Task dependency graph corresponding to the example script in Figure 1.

\begin{tabular}{|l|l|}
\hline Argument & Value \\
\hline Formal & - INOUT: read-write object. \\
parameter & - OUT: write-only object. \\
name & - FILE: read-only file. \\
& - FILE_INOUT: read-write file. \\
& - FILE_OUT: write-only file. \\
\hline returns & $\begin{array}{l}\text { int (for integer and boolean), long, float, } \\
\text { str, dict, list, tuple, user-defined classes. }\end{array}$ \\
\hline isModifier & True (default) or False. \\
\hline priority & True or False (default). \\
\hline
\end{tabular}

Table 1 Arguments of the @task decorator.

script, as exhibited by the graph in Figure 2: the compute_step tasks can be executed in parallel, while the merge tasks cannot. The programmer does not control such parallelisation explicitly; instead, she programs sequentially and provides information about the task parameters and their direction. Actually, the program in Figure 1 can be executed sequentially by using a sequential version of the PyCOMPSs libraries, imported in line 7 of Figure 1(a) and lines 2-3 of Figure 1(b).

\subsection{Syntax}

After discussing a complete PyCOMPSs example in Section 3.1, this section specifies in a more comprehensive way the syntax of the PyCOMPSs programming model.

\subsubsection{Task Definition}

In PyCOMPSs, the user can define as a task:

- Functions.

- Instance methods: methods invoked on objects.

- Class methods: static methods belonging to a class.

PyCOMPSs tasks can be defined by means of the @task decorator, which provides information about the parameters of the function/method and about the task itself. Table 1 summarises all the arguments supported by the decorator.

On the one hand, the metadata corresponding to a parameter is specified as an argument of the decorator, whose name is the formal parameter's name and whose value defines the type and direction of the parameter. Thus, when including parameter metadata in the @task decorator, the user has the options shown in the first row of Table 1 . The parameter types and directions can be:

- Types: primitive types (integer, long, float, boolean), strings, objects (instances of user-defined classes, dictionaries, lists, tuples, complex numbers) and files are supported. PyCOMPSs is able to automatically infer the parameter type for primitive types, strings and objects, but not for files, which need to be defined as FILE.

- Direction: it can be read-only (IN - default), readwrite (INOUT) or write-only (OUT).

Consequently, in the following cases there is no need to include an argument in the Ctask decorator for a given task parameter:

- Parameters of primitive types (integer, long, float, boolean) and strings: the type of these parameters can be automatically inferred, and their direction is always IN.

- Read-only object parameters: the type of the parameter is automatically inferred, and the direction defaults to IN.

On the other hand, there are three reserved arguments of the @task decorator, also presented in Table 1. First, if the function or method returns a value, the programmer must specify the type of that value using the returns argument. Return values can be seen as parameters with OUT direction. Second, for tasks corresponding to instance methods, by default the task is assumed to modify the callee object (the object on which the method is invoked). The programmer can tell otherwise by setting the isModifier argument to False. Finally, the programmer can also mark a task as a high-priority task by setting the priority argument to True. This way, when the task is free of dependencies, it will be scheduled before any of the available low-priority (regular) tasks. This functionality is useful for tasks that are in the critical path of the application's task dependency graph.

Figure 3 shows an example of the usage of file parameters in PyCOMPSs tasks, which can be useful to program computational workflows. The script in Figure 3(a) calls a function func, which receives a string parameter containing a file name. Note that, for PyCOMPSs to know that my_file is actually a file path that must be treated as such - and not as any other string - the user needs to state that the parameter is of type FILE. In addition, since the code of func updates the file, the INOUT direction also needs to be specified. 
my_file = 'sample_file.txt'

func(my_file)

from pycompss.api.task import task

from pycompss.api. parameter import *

$$
\begin{aligned}
& \text { Otask(f }=\text { FILE_INOUT) } \\
& \operatorname{def} \operatorname{func}(\mathrm{f}) \text { : } \\
& \quad \mathrm{fd}=\operatorname{open}\left(\mathrm{f}, \mathrm{\prime}^{\prime} \mathrm{r}+{ }^{\prime}\right)
\end{aligned}
$$$$
\cdots
$$

Fig. 3 Example of a task receiving a file parameter: (a) call to function from the application, (b) decorated function.

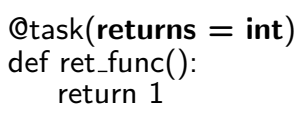

Fig. 4 Definition of a task returning an integer.

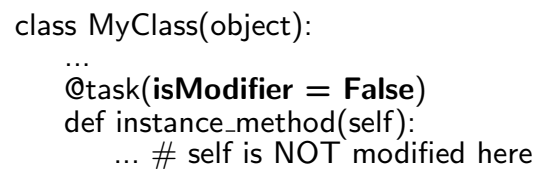

Fig. 5 Example of usage of the isModifier flag.

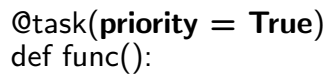

Fig. 6 Example of usage of the priority flag.

Regarding the other arguments of the @task decorator: (i) Figure 4 defines a task that returns an integer value, (ii) Figure 5 shows a usage example of the isModifier argument and (iii) Figure 6 defines a task with priority.

\subsubsection{Main Program}

The main program of the application is a sequential Python script (or scripts) that contains calls to tasks. Tasks can modify or generate data (e.g. a file or object), and these data can eventually be accessed from the main program. Before doing so, however, the programmer needs to synchronise that data (i.e. stall the main control flow until obtaining the last version produced by the task, which can imply waiting for the task to finish). As a result, the main program can work with the correct version of the data.

Depending on the data type that is being synchronised, two API functions may need to be invoked (summarised in Table 2):

- compss_wait_on(obj, to_write $=$ True): synchronises for the last version of object obj and returns the synchronised object. It can receive an optional boolean parameter to_write, which defaults to True, that in-

\begin{tabular}{|l|l|}
\hline Function & Use \\
\hline $\begin{array}{l}\text { compss_wait_on } \\
\text { (obj, to_write }=\text { True) }\end{array}$ & $\begin{array}{l}\text { Synchronises for the last version } \\
\text { of an object and returns it. }\end{array}$ \\
\hline $\begin{array}{l}\text { compss_open } \\
\text { (file_name, mode }=\text { ' } r \text { ') }\end{array}$ & $\begin{array}{l}\text { Synchronises for the last version } \\
\text { of a file and returns its file } \\
\text { descriptor. }\end{array}$ \\
\hline
\end{tabular}

Table 2 PyCOMPSs API functions.

from pycompss.api.api import compss_wait_on,compss_open

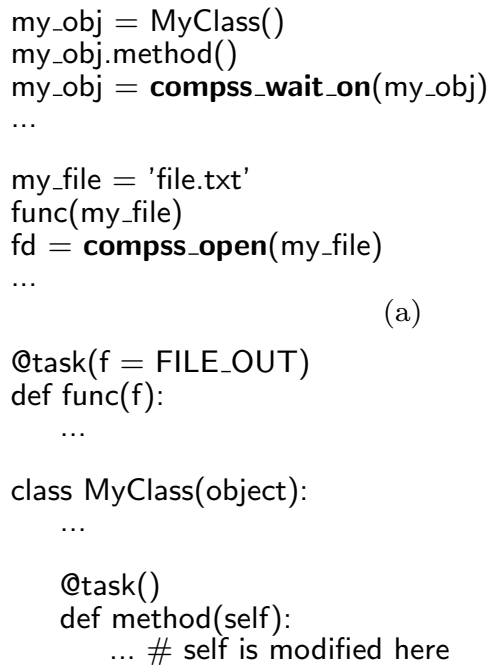

(b)

Fig. 7 Example of synchronisation: (a) main program containing the synchronisation calls, (b) task definition.

dicates whether the main program will modify the returned object.

- compss_open(file_name, mode = ' $r$ '): similar to the Python open() call. It synchronises for the last version of file file_name and returns the file descriptor for that synchronised file. It can receive an optional parameter mode, which defaults to ' $r$ ', containing the mode in which the file will be opened (the open modes are analogous to those of Python open()).

To illustrate the use of the aforementioned API functions, the example in Figure 7 first creates an object of class MyClass and invokes a task method called method that modifies the object; the object is then synchronised with compss_wait_on(), so that it can be used in the main program from that point on. After that, the script invokes a task func that writes a file, which is later synchronised by calling compss_open().

If the programmer defines as a task a function or method that returns a value, that value is not generated until the task executes. However, in order to keep the asynchrony of the task invocation, PyCOMPSs manages future objects [24]: a representant object is immediately returned to the main program when a task is invoked. The future object mechanism is applied to prim- 


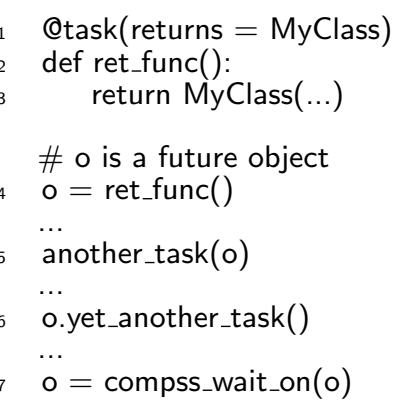

Fig. 8 Future objects in PyCOMPSs.

itive types, strings and objects (including the Python built-in types list, dictionary, tuple and complex).

As shown in Figure 8, a future object returned by a task (object o, line 4) can be involved in subsequent asynchronous task calls (lines 5 and 6 ), and PyCOMPSs will automatically find the corresponding data dependencies. On the other hand, in order to synchronise the future object from the main program, the programmer proceeds in the same way as with any object updated by a task (line 7$)$.

\section{Runtime System}

The programming model described in Section 3 is enabled by a runtime system that parallelises the application on behalf of the user.

This section describes the basic implementation of such runtime system, i.e. how it generates and manages tasks and how it processes the data accessed by those tasks.

\subsection{Architecture}

PyCOMPSs was designed to operate on top of the COMPS $[30,28]$ Java runtime system, acting as a language binding for Python applications. This design decision allowed PyCOMPSs to leverage COMPSs' functionalities and, as a result, require a shorter development time.

Among the functionalities implemented by COMPSs there are data dependency analysis, task scheduling, data transfer and fault tolerance. In addition, it can execute applications on different kinds of distributed infrastructures such as clouds, clusters or grids. On top of the Java runtime, the Python binding handles the computations and data of the application and interacts (through a $\mathrm{C}++$ library) with the Java libraries underneath. Figure 9 shows the architecture of the whole framework.

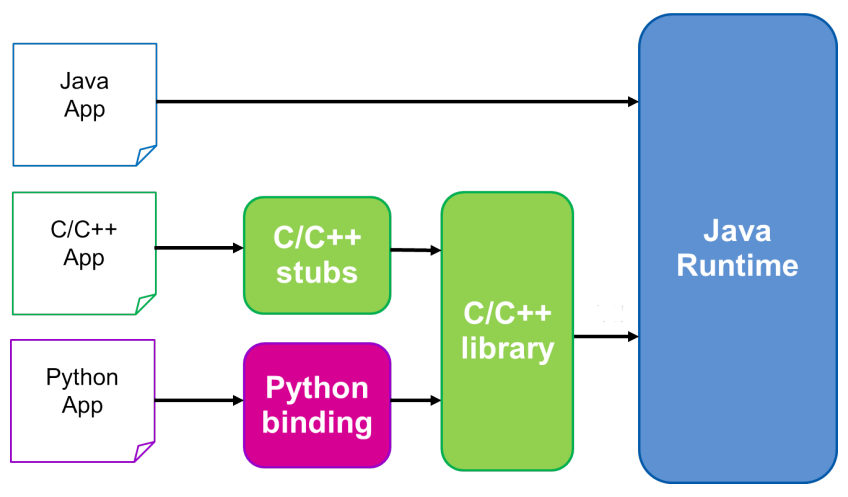

Fig. 9 Architecture of the PyCOMPSs runtime system.

\subsection{Task Generation}

Calls to functions decorated with @task are wrapped by a function of the Python binding, which forwards the function name and parameters to the Java runtime. With that information, the Java runtime creates a task and adds it to the data dependency graph, immediately returning the control to Python. At this point, the main program can continue executing right after the task invocation, possibly invoking more tasks.

Therefore, the Java runtime executes concurrently with the main program of the application, and as the latter issues new task creation requests, the former dynamically builds a task dependency graph. Such graph represents the inherent concurrency of the program, and determines what can be run in parallel. When a task is free of dependencies, it is scheduled by the runtime system on one of the available resources, specified in XML configuration files.

The default scheduling policy of the runtime is localityaware. When scheduling a task, the runtime system computes a score for all the available resources and chooses the one with the highest score. This score is the number of task input parameters that are already s present on that resource, and consequently they do not need to be transferred.

\subsection{Task Offloading and Data Management}

Once a task has been scheduled, and before submitting the task to the target resource for execution, the Java runtime transfers the missing task input data to that resource.

Tasks created by a PyCOMPSs application can receive different types of data as a parameter, as explained in Section 3.2. Specifically, tasks work with data that is either in memory (Python objects, primitive types) or disk (files). Such data needs to be forwarded 


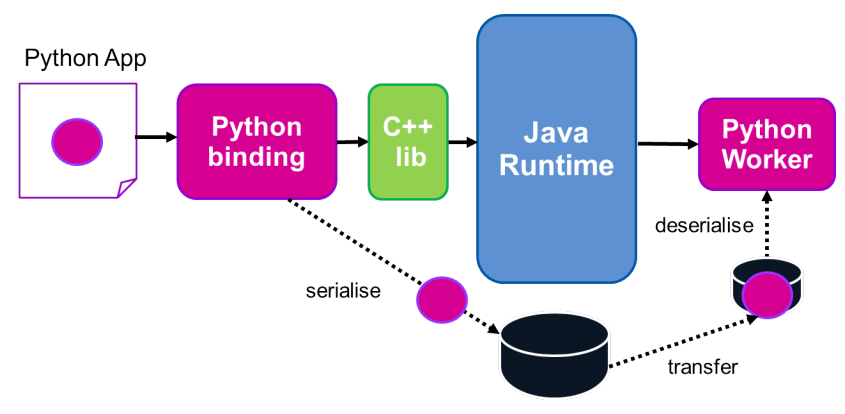

Fig. 10 Management of objects in the basic implementation of the PyCOMPSs framework.

to the Java runtime, which will be in charge of transferring it to the destination resource where the task will run. Primitive types in Python are mapped by the Python binding to the equivalent types in Java, and files are processed as such on the Java side.

Regarding Python objects, their management is a bit more complex. Figure 10 shows how objects are handled by PyCOMPSs. Objects can be created by a Python application in Python space and later be accessed by a task. Since neither these objects nor their associated classes exist in the Java space, they are serialised to files by the Python binding. Hence, when informing the Java runtime of the creation of a new task, the Python binding will define all object parameters as files so that the Java runtime can process and transfer them.

Once the input data for a task is transferred, the Java runtime will trigger the execution of a Python worker script on the worker resource. This script will process the request of running a task, check which data it needs (deserialising the input objects if necessary) and finally run the task.

The runtime processing described in this section obviously comes with an overhead. Therefore, for the remote execution of tasks to be worthwhile, the duration of these tasks should be in the order of tens or hundreds of milliseconds, depending on the number of resources to be used.

\section{Support for Big Data}

Section 4 described the basic implementation of the PyCOMPSs runtime, where tasks operate on data that is either in memory (regular Python objects and primitive types) or disk (files). In order to make PyCOMPSs capable of orchestrating applications that process big amounts of data, the data management of PyCOMPSs was extended both at programming model and runtime system levels.

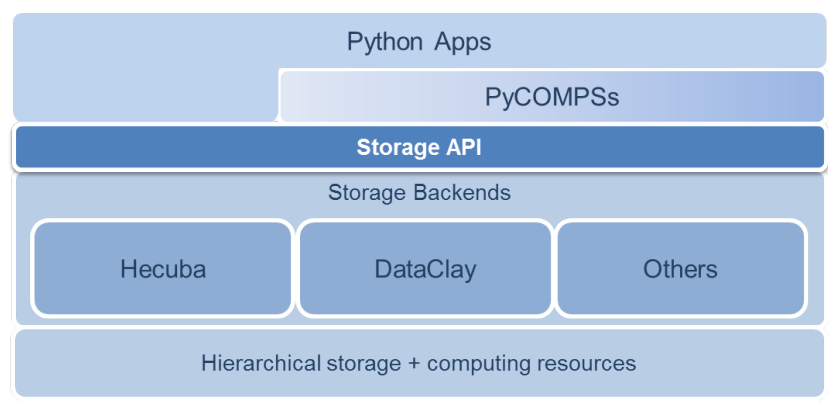

Fig. 11 PyCOMPSs on top of a persistent storage layer.

In that sense, this section presents the work on integrating PyCOMPSs in a persistent object storage platform. Such platform provides PyCOMPSs scripts, as well as the tasks they generate, with a distributed, fault-tolerant and efficient storage layer. Furthermore, multiple applications can share data in a concurrent way through this layer.

The main objective of the aforementioned platform is facilitate the access to data as much as possible. Any function in a Python script, even if it has not been developed with PyCOMPSs in mind, should be able to seamlessly work with objects in memory or with persistent objects, with no need to be adapted for each case. It is the responsibility of the main program, which invokes the function, to decide whether the object instance is made persistent or not depending on the purpose of such object, but this decision is transparent to the function.

Figure 11 depicts the overall structure of the proposed storage platform. Python scripts programmed with the PyCOMPSs model are located at the top of the stack, and the tasks they generate are processed by the PyCOMPSs runtime system. These scripts rely on a Storage API in order to create, delete, insert, retrieve and iterate over persistent data. At its turn, PyCOMPSs also invokes the Storage API, mainly to obtain locality information about persistent data.

The Storage API can be implemented by multiple Storage Backends. A Storage Backend is responsible for storing data in a set of distributed resources, managing data format and organisation and optimising data queries.

The next subsections present the extensions to PyCOMPSs that were required to operate on top of this storage platform, and they also describe a backend that implements the Storage API. 


\subsection{Programming with Persistent Objects}

The data that resides in the storage platform can have multiple formats, depending on the backend that stores it. Nevertheless, from the point of view of the application, such data is abstracted and it is always accessed as Python objects, no matter how it is stored underneath.

Consequently, the programming model proposed here is based on the use of objects. Such objects can be made persistent by an application, that is, objects initially allocated in memory can be backed by the persistent storage layer. From that point on, changes to the object will be forwarded to the backend as well. On the other hand, objects that were made persistent can be retrieved by other applications. This enables interactive sharing of data between applications that run concurrently.

\subsubsection{Managing Persistency}

In order to deal with persistent objects, the storage platform requires some knowledge about the classes that will be stored. In particular, since Python is a dynamically typed programming language, the names and types of the attributes are not included in the class definition, and they should be specified to correctly map the class representation to the backend. This can be done by means of docstrings, the standard Python documentation mechanism, as shown in figure 12(a), line 2. This would be then used to generate the corresponding code that implements the Storage API.

To make an object persistent, the programmer needs to invoke the make_persistent method, which is part of the Storage API. Figure 12(b) shows a simple example where two regular Python objects o1 and o2 of class Foo are created (lines 4-5). After that, make_persistent is invoked on o1 in order to be stored as persistent (line 6 ). The make_persistent method receives a parameter of type string that specifies the alias - a name or identifier - that the user wants to give to that object. Such alias must be unique in the storage namespace, and it can be used afterwards to retrieve the object.

A persistent object can be involved in a task call like any regular (non-persistent) object, as exemplified in line 7 of Figure 12(b), and the programmer does not need to provide any additional information in the @task decorator (line 1). my_func can be a pre-existing function, completely unaware of object persistency. Thus, it manipulates the persistent object o1 and the regular object o2 in the same way (line 3 ). However, in the case of o1, any changes to the object are transparently persisted in the distributed storage. Also importantly, the

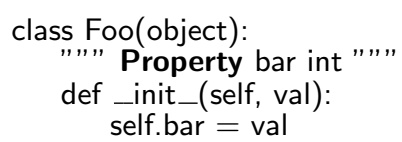

(c)

Fig. 12 Managing persistent objects: (a) class definition, (b) producer script that makes an object persistent, (c) consumer script that loads a persistent object.

\begin{tabular}{|l|l|}
\hline Method & Use \\
\hline make_persistent & Stores an object \\
\hline delete_persistent & $\begin{array}{l}\text { Deletes an object from } \\
\text { persistent storage }\end{array}$ \\
\hline Constructor('alias') & $\begin{array}{l}\text { Retrieves the persistent object } \\
\text { with the alias provided }\end{array}$ \\
\hline
\end{tabular}

Table 3 Storage API for applications.

PyCOMPSs runtime will try to schedule the task on the resource where the object is stored to favour locality.

Figure 12(c) depicts a small consumer script that loads the same object that was stored in Figure 12(b). In order to do that, the script invokes a constructor of class Foo that receives the alias of the object as parameter (line 3). This constructor is provided by the implementation of the model, and it returns a reference to the desired persistent object. Once the reference is obtained, the object can also participate in a task call (line 4).

Table 3 details the methods included in the Storage API that provide applications with the ability to manage persistent objects.

\subsubsection{Persistent Dictionaries}

A particular case when working with persistent objects is that of dictionaries. The Python dictionary built-in type is an unordered set of key-value pairs. Keys can be of any immutable type, including tuples. Dictionaries 


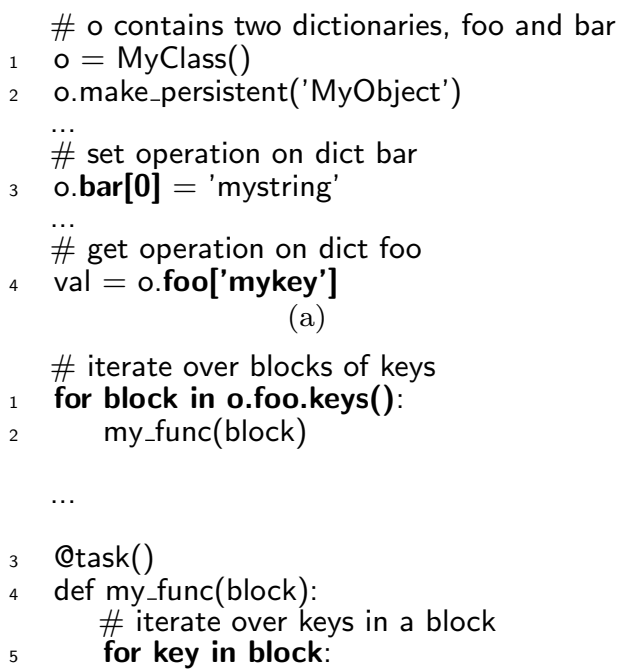

Fig. 13 Managing persistent dictionaries: (a) getting and setting elements, (b) iterating over keys.

(or objects containing dictionaries) can be made persistent and, when that happens, the data they contain can be easily mapped to a key-value store in a Storage Backend.

In that sense, a PyCOMPSs application can manipulate a persistent dictionary as it would do with any regular Python dictionary, e.g. adding a new key-value pair or requesting the value associated to a given key. Since the dictionary is persistent, any operation will be translated into an access to the persistent data. In Figure 13(a), an object o of class MyClass is instantiated in line 1 and made persistent in line 2. Let us assume that object o has two attributes of type dictionary, foo and bar. Even if o is persistent, the programmer can add new entries to its dictionaries (line 3) or get the value for a given key (line 4) in a normal way. In the case of a persistent object, though, such operations will be forwarded to the Storage Backend, e.g. a distributed key-value store.

On the other hand, Python allows to retrieve the set of keys in a dictionary by invoking the keys() method on it. In addition, these keys can be iterated to execute some operation for each key-value pair. In a persistent dictionary, obtaining the keys and iterating over them can be done like with a regular dictionary, but the semantics of these operations differs from the original one.

As can be seen in Figure 13(b), when iterating over the keys of a persistent dictionary (line 1), the iterator will return blocks of keys instead of individual keys. Those blocks correspond to the partitions of key-value pairs in the underlying Storage Backend. This feature is leveraged to exploit data locality: PyCOMPSs tasks that receive one or more blocks as parameters can be created (line 2), and the PyCOMPSs runtime will try to schedule them in the resource where those blocks reside. If that happens, any access to the keys in that block will be local. For instance, if such block is iterated inside the task to obtain the keys it holds (line 5), the keys (and their associated values) will already be on the resource that runs the task.

\subsection{Storage API and Backends}

The Storage API lies underneath the application and the PyCOMPSs runtime (Figure 11) and offers methods to access persistent objects and obtain information about them.

Section 5.1 introduced those methods that the Storage API provides to manage objects from applications, i.e. to make objects persistent, to load them or to modify their attributes. In addition to these methods, the Storage API also provides a set of methods that enable PyCOMPSs to take into account data locality when scheduling tasks with persistent objects as parameters. In order to do that, the PyCOMPSs runtime invokes the getLocations method of the Storage API, which returns the resources where a given persistent object or block is stored. With this information, PyCOMPSs can try to submit a task to a resource where (at least part of) its input data is already present, thus preventing remote accesses to the data from the task. This method receives as a parameter the identifier of an object, which is assigned by the storage platform. This identifier can be obtained by means of the method getID. Finally, the method getByID provides a reference to the persistent object with the identifier specified as a parameter.

The methods of the Storage API can be implemented by one or more Storage Backends, which transform the generic operations on persistent objects into specific operations on their associated backend data. The next subsection describes the Storage Backend that has been developed so far for its use in the Storage Platform.

\subsubsection{Hecuba}

Hecuba is a set of tools and interfaces developed in our research group, that aims to facilitate programmers an efficient and easy interaction with non-relational databases. In particular, we have added to Hecuba the implementation of the interface necessary to provide PyCOMPSs with a Storage Backend suitable to support Big Data applications. Currently, this interface is implemented on Apache Cassandra database, although it is easy to port this implementation to any non-relational key-value data store. 
Cassandra [1] is a distributed and highly scalable key-value database. Cassandra implements a non-centralized architecture, based on peer-to-peer communication, in which all nodes of the cluster are able to receive and serve queries. Each node of the cluster is assigned a token. A partitioner function uses this token to decide how data is distributed among the nodes in the shape of a ring.

Data in Cassandra is stored on tables by rows, which are identified by a key chosen by the user. This key can be composed by one or several attributes. In each row the user can add additional attributes also identified by a name. In order to enhance data locality when accessing data stored in Cassandra, it is necessary to understand how is data organized. Cassandra stores data by rows, and one node is responsible for hosting a specific row. The target node is chosen based on the key of the row and of the token of each node by the partitioner algorithm. In order to guarantee data availability, Cassandra can be configured to keep several replicas for each data. In a setup with $\mathrm{N}$ replicas it is also necessary to choose the nodes that will hold each replica. The default replication algorithm selects the $\mathrm{N}-1$ nodes that come after the target node in the ring's order to store those replicas.

The mapping of a Python dictionary on a data model in Cassandra is straightforward as both consist on values indexed by keys. Thus, the implementation of a Persistent Dictionary backed up by Hecuba is straightforward too. We have decided to map each class containing one or more Persistent Dictionary on a Cassandra table. This table is indexed by the same key attributes than the Persistent Dictionaries in the class, and contains as many non-key attributes as Persistent Dictionaries. In the current implementation Hecuba only supports the backing of Persistent Dictionaries but as part of our future work we plan to extend it to support other type of attributes.

Following with the example described in section 5.1.2, we will show how to implement a class backed up by Hecuba. For the sake of rapid prototyping, our current implementation has some variations regarding the definition of classes explained in section 5.1.1. In particular, figure 15 shows the minimum code required to implement a class containing two Persistent Dictionaries. It is just necessary to indicate that the class is a subclass of StorageObj, which is a class implemented inside Hecuba, and that contains all the methods that are necessary to access and manipulate data backed up by Cassandra. Thus, the code of the application is mostly independent of the data backend used. Notice that programmers can add their own methods to the class definition, the only requirement is that the constructor of the StorageObj

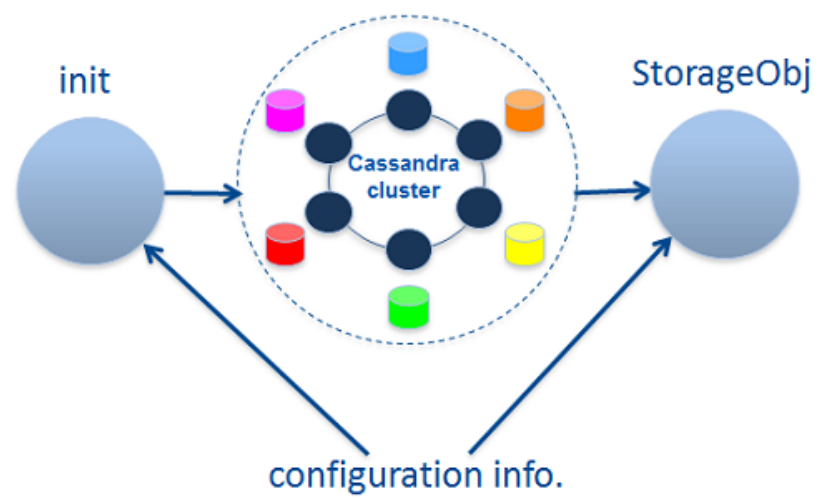

Fig. 14 Configuration of StorageObj.

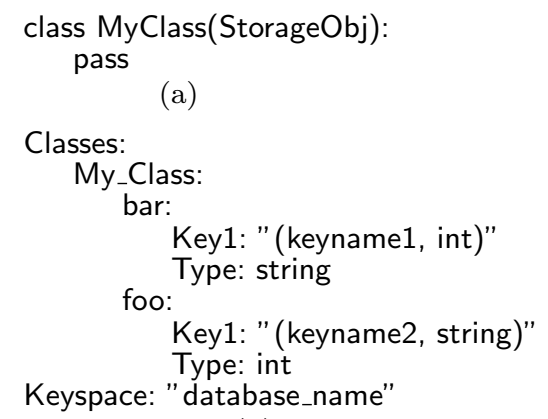

Fig. 15 Defining a user class backed by Hecuba: (a) minimum class code, (b) data scheme definition.

class has to be executed each time a new object of the class is instantiated.

In addition to this simple class definition, users have to provide the information necessary to connect to the Cassandra cluster (ip addresses and ports) and to configure the database. Configuration of the database requires information about the database identifier (keyspace name) and the data scheme (for each table, name and type of each attribute and which attribute is part of the key). Information about the Cassandra cluster can be set through environment variables and it is common to all applications executed on this cluster. Information about the database configuration can be codified in a yaml file. Figure 15 represents the yaml file that the user should provide to complete the configuration of the application. During the initialization phase of the application, Hecuba code reads and parses this yaml file and configures itself to be able to adapt at runtime the generic code of the StorageObj methods to the particular data schemes defined (see figure 14).

Following we describe the implementation of the interface implemented by Hecuba as part of the Stor- 
ageObj class. The methods exported to programmers allow to perform the following tasks:

- Make an object persistent: this operation is performed through the make_persistent method of the StorageObj. The implementation of this method creates the Cassandra table that it is necessary to hold the object and populates it with the data that the object had in memory.

- Object instantiation: it is implemented by the constructor of the StorageObj class. If the constructor receives a parameter, the constructor binds the instantiated object with the corresponding Cassandra table. This way, all the following accesses to that object will be translated into accesses to that table. On the contrary, if the constructor does not receive a parameter, all data associated to that new object instance will be kept in memory until the makePersistent method is executed.

- Query/Update data: these operations are implemented through the get_item and the set_item methods of the Persistent Dictionaries. There are two different scenarios: if the object is in memory, the implementations of these methods are translated into the usual methods of a Python dictionary; if the object is backed by a Cassandra table, then the implementations of these methods consist on queries performed on the Cassandra table.

- Data iteration: as we have explained in section 5.1.2, the implementation of the keys method of a Persistent Dictionary returns a block of keys that will be the input parameter of a task. In order to enhance data locality, we decided to create the blocks of keys based on their node location. This way, PyCOMPSs can use the information about the data location to schedule tasks local to the data. Thus, we have implemented two different iterators: one of them to create the different blocks of keys and the other one to traverse all the keys in a block (see figure 16).

- Delete a persistent object: this operation is implemented by the delete_persistent method of the StorageObj and it just deletes from the database the table that was backing the object.

\section{Evaluation}

This paper presents the evaluation of PyCOMPSs in two different cases: evaluation of PyCOMPSs standalone and evaluation of PyCOMPSs with the support for Big Data with the Hecuba backend.

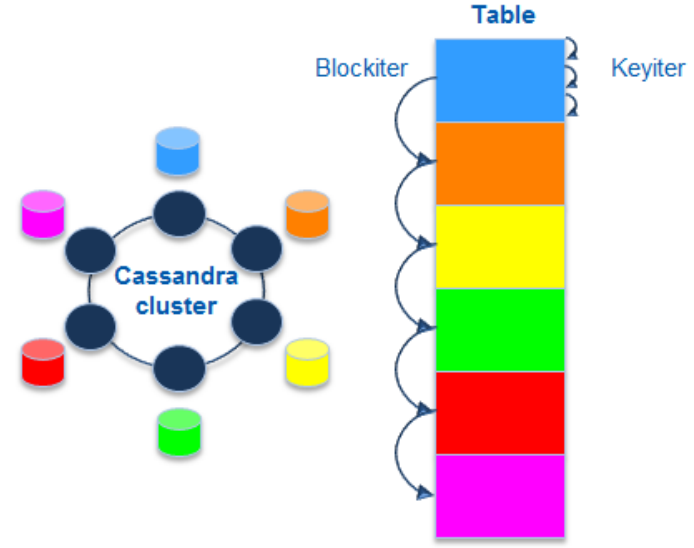

Fig. 16 Implementation of iterators.

\subsection{Test Environment}

The evaluation has been performed in two different environments. The standalone PyCOMPSs tests have been executed in the MareNostrum III supercomputer. MareNostrum III is a cluster with 3,056 compute nodes, each of them 2x Intel SandyBridge-EP E5-2670/1600 $20 \mathrm{M}$ with 8 cores at $2.6 \mathrm{GHz}$ connected with a Infiniband FDR10 network.

The tests of PyCOMPSs with the support for Big Data have been performed in a cluster of 5 nodes. Each node is equipped with 2 Intel Xeon Quad-Core L5630 at $2.13 \mathrm{GHz}, 24 \mathrm{~GB}$ RAM and 6 TB HDD, and they are interconnected with Gigabit Ethernet. Out of the five nodes, one executes the main program of the application and the PyCOMPSs master runtime, while the rest are PyCOMPSs worker nodes that execute tasks. The 4 worker nodes also form a Cassandra ring and have the Hecuba backend installed. The master node has an Hecuba client and a Cassandra client.

In all cases we used PyCOMPSs version $1.2^{1}$.

\subsection{Standalone Tests}

PyCOMPSs has been evaluated in MareNostrum III with two different applications: DimSweep and NeuronCorr. DimSweep performs a cluster architecture exploration using the Dimemas simulator performing a parameter sweep of several configuration values: Fabric Interconnection Network latency and bandwidth, number of nodes, CPU speed, and intranode latency and bandwidth.

Dimemas [27] is a simulator of the behavior of MPI applications under different network and architecture

\footnotetext{
1 Available at www.bsc.es/compss
} 


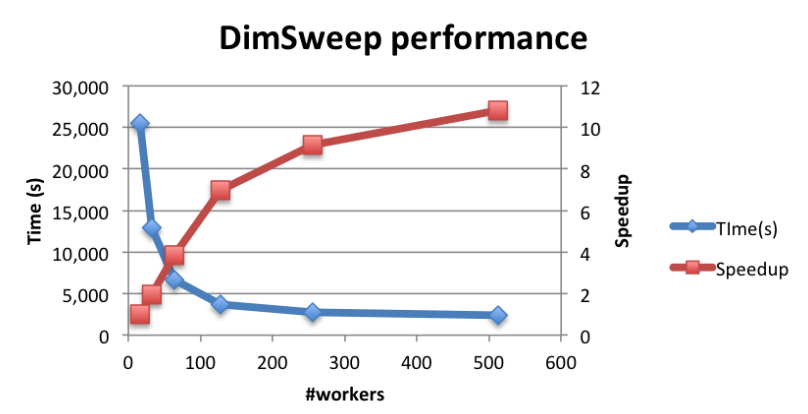

Fig. 17 Performance of DimSweep. The chart shows ellapsed time and speed-up using as baseline the 16 workers case.

conditions by means of replaying the applications' executions recorded in traces. This PyCOMPSs example is codified with two different tasks' types: one that executes the Dimemas simulation and another one that accumulates the results. While the simulation tasks are independent between them, the accumulation tasks create a chain of dependencies that serialize all them. To avoid a long chain of these tasks at the end of the execution, these tasks are prioritized in such a way that are inserted between the simulation tasks.

For the experiment a case with a real execution tracefile and a combination of parameters' configurations that generated 2304 tasks was used. Figure 17 shows the results obtained when varying the number of cores used for PyCOMPSs workers from 16 to 512 . The time is the total ellapsed time and the speedup is computed using as baseline the case with 16 workers. The results show very good scaling up to 128 workers, reducing a bit for larger processor counts.

NeuronCorr is a neuroscience data processing example that computes all mutual cross-correlations between all pairs of a set of spike data [23]. The original example was written in Parallel Python and has been translated to PyCOMPSs. The example has two tasks' types: one that compute the cross-correlations for a block of data and a second one that gathers the results in a data structure. Since the gathers create a chain of tasks, this type of task is prioritized to avoid a long serial chain at the end of the execution. The evaluation was performed with a data set that generates 2048 tasks.

Figure 18 shows the results obtained for the NeuronCorr example. Due to the small granularity of the tasks this example does not scale as well as the DimSweep one.

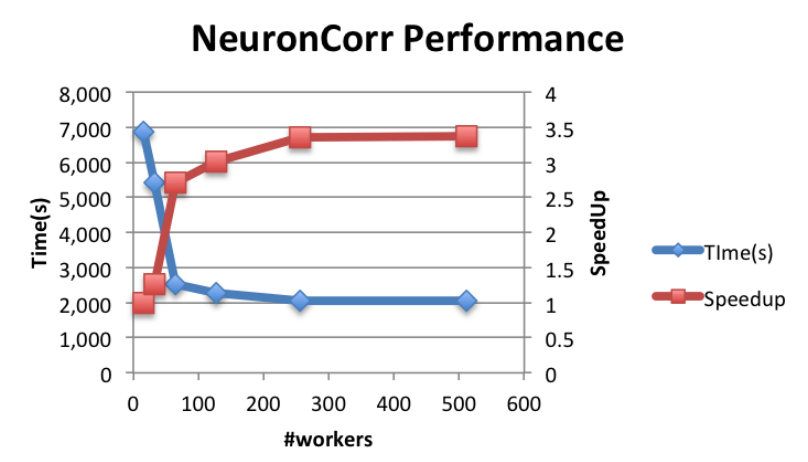

Fig. 18 Performance of NeuronCorr. The chart shows ellapsed time and speed-up using as baseline the 16 workers case.

\begin{tabular}{|l|l|r|}
\hline Cassandra topology & PyCOMPSs workers & Time(s) \\
\hline $4,4,0,0$ & $4,4,0,0$ & 19228 \\
\hline $8,8,0,0$ & $8,8,0,0$ & 10273 \\
\hline $16,16,0,0$ & $16,16,0,0 *$ & 12867 \\
\hline $16,16,16,16$ & $16,16,16,16 *$ & 6644 \\
\hline
\end{tabular}

Table 4 Performance results for the NeuronCorr application with persistent storage for some configurations. The column on the left describes the Cassandra configuration, indicating how the Cassandra nodes are mapped in the Minerva cluster nodes. The column in the middle describes the number of PyCOMPSs workers mapped in each of the Minerva cluster nodes. In the cases marked with $*$, multithreading is activated.

\subsection{Tests on Persistent Storage}

For this section, PyCOMPSs has been evaluated with NeuronCorr application, in the Minerva cluster (Figure 19). All simulation tasks are independent, and input data is obtained from a Cassandra table previously created. Once the execution starts, input data is structured in blocks, and this information is used by tasks to know which Cassandra node contains its information. Once each task has finished executing, resulting data is saved in Cassandra.

Table 4 presents results using different configurations of Cassandra nodes and PyCOMPSs workers for a given input data. The column in the left shows the mapping of the Cassandra nodes to Minerva cluster nodes. The column in the middle shows the mapping of PyCOMPSs workers to the Minerva cluster nodes, using multithreading in some cases (marked with $*$ ). The same total count of Cassandra nodes and PyCOMPSs workers is used to balance workload. These results show good scaling between the cases of the same nature (with multithreading or without multithreading). The results also reflect the impact of sharing resources between threads when multithreading is used. 


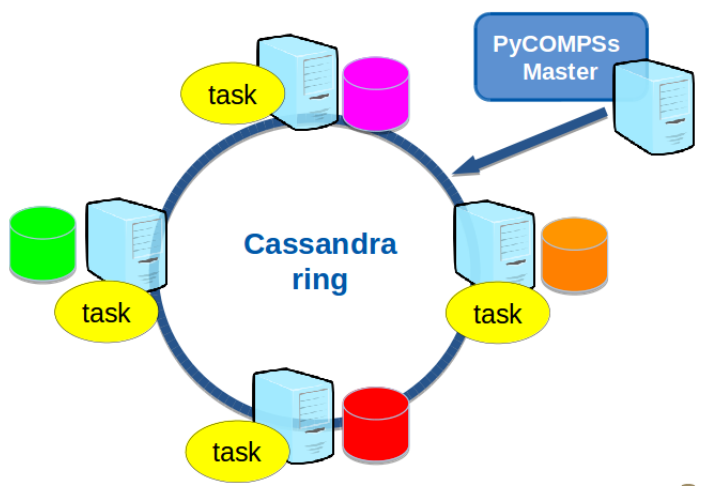

Fig. 19 Hecuba Topology for Persistent Storage

\section{Conclusions and Future Work}

This paper has presented PyCOMPSs, a programming framework that facilitates the development of parallel computational workflows in Python. With PyCOMPSs, the user programs her script in a sequential fashion and decorates the functions to be run as asynchronous parallel tasks. A runtime system is in charge of exploiting the inherent concurrency of the script, detecting the data dependencies between tasks and spawning them to a set of distributed resources. Those resources can be physical nodes in a cluster or grid or virtual machines in a cloud.

We have also described an extension to the PyCOMPSs programming model and runtime system that builds on top of a persistent object storage layer. In this approach, the application data is stored in a distributed storage backend (e.g. a key-value store like Cassandra). From the application point of view, such data is abstracted and accessed as Python objects, no matter the particular backend being used underneath. PyCOMPSs tasks can receive persistent objects as parameters, and these tasks will be scheduled taking into account data locality, launching them to resources that store the data they need. Furthermore, multiple applications can share data in a concurrent way through this persistent object layer.

The future work includes incorporating a new backend for persistent objects, based on the Scalable KeyValue Store [16] being developed at IBM. On the other hand, we will offer a web portal for programming, deploying and executing PyCOMPSs scripts on the cloud, which will leverage the IPython notebook [6] tool as the main interface for interactive script development and execution.

Acknowledgements This work has been supported by the following institutions: the Spanish Government with grant SEV-2011-00067 of Severo Ochoa Program and contract Com- putación de Altas Prestaciones VI (TIN2012-34557); by the SGR programme (2014-SGR-1051) of the Catalan Government; by the project The Human Brain Project, funded by the European Commission under contract 604102; and by the Intel-BSC Exascale Lab collaboration.

\section{References}

1. Apache cassandra. http://cassandra.apache.org/.

2. Celery: Distributed task queue. http://www.celeryproject.org.

3. Decorators for Functions and Methods. https://www.python.org/dev/peps/pep-0318/.

4. dispy: Python framework for distributed and parallel computing. http://dispy.sourceforge.net.

5. Flow based programming. https://wiki.python.org/moin/FlowBasedProgramming.

6. IPython Notebook. http://ipython.org/notebook.html.

7. matplotlib. http://matplotlib.org/.

8. Matrix Laboratory http://www.mathworks.com/products/matlab/.

9. NumPy. http://www.numpy.org/.

10. pandas. http://pandas.pydata.org/.

11. Parallel Processing and Multiprocessing in Python. https://wiki.python.org/moin/ParallelProcessing.

12. Parallel Python http://www.parallelpython.com.

13. pyMPI: Putting the py in MPI. http://pympi.sourceforge.net.

14. Python multiprocessing module. https://docs.python.org/2/library/multiprocessing.html.

15. Python programming language. https://www.python.org/.

16. Scalable Key-Value Store. https://github.com/ScalableKey-Value.

17. SciPy. http://www.scipy.org/.

18. Sqlalchemy. http://www.sqlalchemy.org/.

19. Sqlobject. http://sqlobject.org/.

20. Zodb object database. http://www.zodb.org.

21. R. M. Badia, J. Labarta, R. Sirvent, J. M. Pérez, J. M. Cela, and R. Grima. Programming grid applications with grid superscalar. Journal of Grid Computing, 1(2):151170, 2003.

22. L. Dalcn, R. Paz, M. Storti, and J. DEla. $\{$ MPI $\}$ for python: Performance improvements and mpi-2 extensions. Journal of Parallel and Distributed Computing, 68(5):655 - 662, 2008.

23. M. Denker, B. Wiebelt, D. Fliegner, M. Diesmann, and A. Morrison. Practically trivial parallel data processing in a neuroscience laboratory. In S. Grn and S. Rotter, editors, Analysis of Parallel Spike Trains, volume 7 of Springer Series in Computational Neuroscience, pages 413-436. Springer US, 2010.

24. Edward F. Walker and Richard Floyd and Paul Neves. Asynchronous Remote Operation Execution in Distributed Systems. In 10th Intl. Conf. on Distributed Computing Systems (ICDCS-10), pages 253-259, May 1990.

25. E. Gafni, L. J. Luquette, A. K. Lancaster, J. B. Hawkins, J.-Y. Jung, Y. Souilmi, D. P. Wall, and P. J. Tonellato. Cosmos: Python library for massively parallel workflows. Bioinformatics, page btu385, 2014.

26. L. Goodstadt. Ruffus: a lightweight python library for computational pipelines. Bioinformatics, 26(21):2778$2779,2010$. 
27. J. Labarta, S. Girona, V. Pillet, T. Cortes, and L. Gregoris. DiP: A parallel program development environment. In L. Bouge, P. Fraigniaud, A. Mignotte, and Y. Robert, editors, Euro-Par'96 Parallel Processing, volume 1124 of Lecture Notes in Computer Science, pages 665-674. Springer Berlin / Heidelberg, 1996. 10.1007/BFb0024763.

28. F. Lordan, E. Tejedor, J. Ejarque, R. Rafanell, J. lvarez, F. Marozzo, D. Lezzi, R. Sirvent, D. Talia, and R. Badia. Servicess: An interoperable programming framework for the cloud. Journal of Grid Computing, 12(1):67-91, 2014.

29. N. Singh, L.-M. Browne, and R. Butler. Parallel astronomical data processing with python: Recipes for multicore machines. Astronomy and Computing, 2:1-10, 2013.

30. E. Tejedor and R. M. Badia. COMP Superscalar: Bringing GRID Superscalar and GCM Together. In Eighth IEEE International Symposium on Cluster Computing and the Grid, CCGrid '08, Lyon, France, pages 185-193, May 2008.

31. Y. Zhao, M. Hategan, B. Clifford, I. Foster, G. Von Laszewski, V. Nefedova, I. Raicu, T. StefPraun, and M. Wilde. Swift: Fast, reliable, loosely coupled parallel computation. In Services, 2007 IEEE Congress on, pages 199-206. IEEE, 2007. 